\title{
Mechanical Properties of Remote-Laser Cut CFRP and Thermographic Laser-Process Monitoring
}

\author{
Michael Rose ${ }^{1,2^{*}}$, Sebastian Schettler ${ }^{1,2}$, Florian Klemm ${ }^{1}$, \\ Eckhard Beyer ${ }^{3}$, Martina Zimmermann1,2 \\ ${ }^{1}$ Technische Universität Dresden, Institute of Materials Science, Dresden, Germany \\ ${ }^{2}$ Fraunhofer Institute for Material and Beam Technology, Dresden, Germany \\ ${ }^{3}$ Technische Universität Dresden, Institute of Manufacturing Science and Engineering, Dresden, Germany \\ Email: *michael.rose3@tu-dresden.de
}

How to cite this paper: Rose, M., Schettler, S., Klemm, F., Beyer, E. and Zimmermann, M. (2020) Mechanical Properties of Remote-Laser Cut CFRP and Thermographic Laser-Process Monitoring. Materials Sciences and Applications, 11, 560-575.

https://doi.org/10.4236/msa.2020.118037

Received: June 19, 2020

Accepted: August 11, 2020

Published: August 14, 2020

Copyright $\odot 2020$ by author(s) and Scientific Research Publishing Inc. This work is licensed under the Creative Commons Attribution International License (CC BY 4.0).

http://creativecommons.org/licenses/by/4.0/

\begin{abstract}
Remote-laser beam cutting is a productive technology without tool wear. Especially when cutting carbon fiber reinforced polymers (CFRP), it offers constant manufacturing quality. Since it is a thermal process, a heat-affected zone (HAZ) is formed at the edge of the cut. Based on quasi-static and cyclic mechanical tests on open-hole specimens, the influence of the process on the mechanical properties of CFRP is shown. The quasi-static tests are in good correlation with results from other researchers by indicating an increase in the maximum tensile stress of the test specimens, cut by remote-laser. The reason is the rearrangement of the shear stresses and a reduction of the notch stress concentration. However, the results of the present study show that excessive expansion of the HAZ leads to a reduction in the maximum tensile stress compared to milled test specimens. Under cyclic load conditions, remote-laser beam cutting does not lead to a more pronounced degradation than milling. The mechanical properties of the notched test pieces are sensitive to the expansion of the HAZ. For the production of components it is therefore necessary that the remote-laser beam cutting is carried out under controlled and documentable conditions. For this purpose, process thermography was tested as a tool for quality assurance. The results show that the technology is basically suitable for this task.
\end{abstract}

\section{Keywords}

CFRP, Remote Laser Cutting, Fatigue, Process Thermography, Process Monitoring 


\section{Introduction}

Remote-laser beam cutting is a contactless and force-free process. This is an advantage when processing CFRP, with its hard and abrasive fibers. There is no tool wear, which results in a constant and high quality of the cut edges. Compared to gas-assisted laser beam cutting, higher cutting edge qualities can be produced. Concretely, this means that a smaller expansion of the HAZ at the edge of the cut can be achieved. The reason for this is the material removal by repeated laser exposures of the cutting contour at very high spot velocities. This ensures a short interaction time between the material and the laser radiation, which reduces the expansion of the HAZ [1].

Various publications have shown that compared to mechanical processing laser beam cutting of CFRP and the presence of a HAZ reduces its quasi-static strength [2] [3] [4]. Also, the fatigue strength decreases slightly in the presence of an HAZ compared to mechanical processing methods [2]. However, it is remarkable that with increasing HAZ the scatter of the number of load cycles endured decreases [5]. Zaeh, Byrne and Stock were able to show that the presence of laser cut notches in the form of holes can be advantageous [6]. Within the process window they investigated, the mean number of load cycles endured increased with increasing width of the HAZ. The reason is the local redistribution of shear stresses due to the evaporation of the matrix at the cut edge. This results in a reduction of the notch stress concentration of the tensile stresses in the fibers aligned in the direction of loading [6] [7].

The current state of research shows that laser beam cutting has an influence on the mechanical properties of CFRP components. The reason is the formation of an HAZ, which expansion depends on the laser cutting parameters [8]. The adjustment of the laser cutting parameters can in turn influence the mechanical properties.

The aim of this study therefore is to close the link between laser cutting parameters, HAZ expansion and the resulting mechanical properties for the processing with continuous emitting high brilliant beam sources. The dependence of the mechanical material properties on the cutting parameters laser power and spot velocity shows the necessity of process control. The present work therefore addresses two approaches to apply thermography as process monitoring tool.

The determination of the expansion of the thermal damage at the edge of the cut was carried out by analyzing a large number of cutting tests with varying cutting parameters accompanied by microscopic cross-sectional images, allowing establishing a direct correlation between process parameters and HAZ expansion. For the mechanical tests, open-hole specimens were used to further investigate the beneficial effects of laser beam cutting on notched components. This could actually be reproduced under quasistatic load conditions. Beyond that, it was shown that the use of laser cutting parameters, which lead to a pronounced thermal damage at the cutting edge, however, reverses this positive effect. No adverse effects on the CFRP have been observed also under cyclic load 
conditions with suitable processing parameters.

\section{Material and Methods}

The examined CFRP was made from prepregs and consolidated in a press. The prepregs are of the type Sigrapreg C U150-0/NF-340/38\% and the pressure was $39.2 \mathrm{~N} / \mathrm{cm}^{2}$. The curing cycle started at room temperature with heating up to $110^{\circ} \mathrm{C}$ within $3 \mathrm{~h}$. Subsequently, re-cooling took place within $13 \mathrm{~h}$. The fibers are of the type Toray T700 with a filament number of $12 \mathrm{~K}$. The bidirectional layup sequence of the CFRP is $\left.[0 / 90)_{3}\right]_{s}$, resulting in a thickness of $1.9 \mathrm{~mm}$. The fiber volume content of $54 \%$ was measured by a wet-chemical determination according to DIN EN ISO 1183-1. The type of matrix polymer is an epoxy.

For the quasi-static and cyclic mechanical testing of the CFRP open-hole specimens, a servo-hydraulic testing device Instron 8501 was used together with a $100 \mathrm{kN}$ load cell. The testing speed for the quasi-static tests was $2 \mathrm{~mm} / \mathrm{min}$. The cyclic fatigue of the specimens was performed with a pulsating stress ratio of $\mathrm{R}=$ 0.1 and a frequency of $10 \mathrm{~Hz}$. Using a thermographic camera of the type InfraTec ImageIR 8300, it was shown for all stress horizons that the surface temperature of the test specimens remains significantly below the glass transition temperature of $\mathrm{T}_{\mathrm{G}}=140^{\circ} \mathrm{C}$ for this frequency. The tests were carried out in the high cycle fatigue range, which is why the number of load cycles is limited to $10^{6}$. The geometry of the test specimen in accordance with the standard ASTM D-7566/D-5766M-11 is shown in Figure 1. The strain detection was realized by a tactile dynamic extensometer Instron 2620-602 with a measuring length of $25 \mathrm{~mm}$. Its contacts with a width of $3 \mathrm{~mm}$ are narrower than the bore diameter of $4.8 \mathrm{~mm}$, so that they are not affected by the typical inter-fiber breaks documented in the literature [9]. The outer, rectangular contour of all of the samples is milled, whereas the open holes are produced by the various cutting strategies for the investigations. All of the specified mechanical stresses refer to the smallest cross section of the specimens at the center of the open hole. The data are nominal mechanical stresses, without consideration of notch stress concentrations.

Since it allows a phenomenological in-situ measurement of the degradation, the relative residual stiffness was determined during the fatigue tests. It is calculated by the equation

$$
\frac{S_{R}}{S_{0}}=\frac{\Delta l_{u, 0}-\Delta l_{l, 0}}{\Delta l_{u, R}-\Delta l_{l, R}} * \frac{F_{u, R}-F_{l, R}}{F_{u, 0}-F_{l, 0}} .
$$

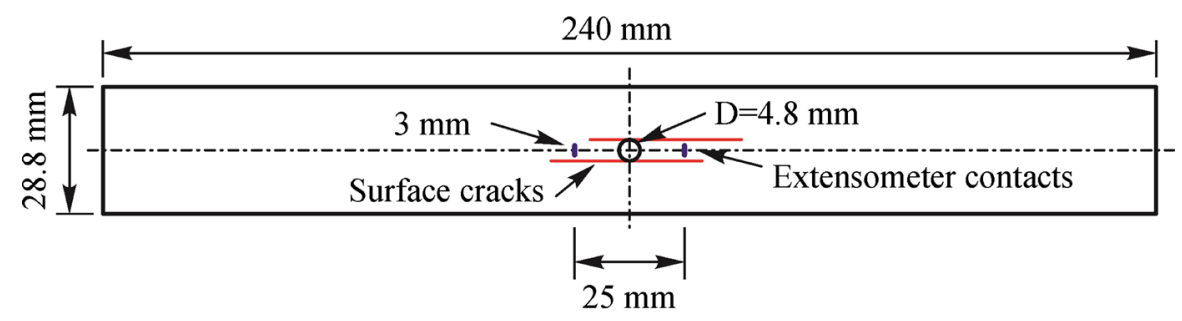

Figure 1. Open hole CFRP specimen geometry according to ASTM D-7566/D-5766M-11. 
The values $\Delta l_{u, 0}$ and $\Delta l_{u, R}$ are the measured displacements of the extensometer for the upper load limit. The index $\mathrm{R}$ means the current value, while index 0 stands for the initial reference. $\Delta l_{l, 0}$ and $\Delta l_{l, R}$ are the displacement changes measured by the extensometer for the lower load limit. The referred initial stiffness $S_{0}$ is the highest one measured during cyclic fatigue. The very first load cycle is also performed with a testing speed of $2 \mathrm{~mm} / \mathrm{min}$ to determine the initial specimen properties under quasi-static conditions as it is suggested in ASTM D-7615/7615M-19. At the very beginning of the tests, the servo-hydraulic testing machine must start oscillating from standstill. The initial load corresponds to the center load. In a first half cycle, the specimen is unloaded to start the oscillation of the machine. The subsequent full cycle is counted as the first one that reveals the highest, initial stiffness $S_{0}$, since the stiffness of the visco-elastic CFRP depends on the testing speed.

For the remote-laser cutting tests and the preparation of the mechanical samples, two different systems with continuous emitting beam sources were used. The specifications can be found in Table 1 .

The remote-laser parameter sets for the processing of the open-hole specimens are summarized in Table 2.

A thermal camera of the type InfraTec ImageIR 8300 was used for thermographic imaging of the remote-laser cutting process. The resolution of $640 \times 480$ pixels allows a maximum recording frequency of $80 \mathrm{~Hz}$. Table 3 gives the emission coefficient of the material. These characteristics were measured by heating of the CFRP. One hour after the laboratory oven reached temperature stability

Table 1. Specification of the used continuous emitting remote-laser systems.

\begin{tabular}{ccc}
\hline & System I & System II \\
\hline Laser & IPG YLS 5000 SM & Rofin DC 035 \\
Scanner system & CTI Lightning II & ScanLab PowerScan 50 \\
Beam source type & Yb:YAG fiber & CO $_{2}$ Slab \\
Intensity distribution & Single mode & Gaussian \\
Nominal output power $P_{\max }$ & $5000 \mathrm{~W}$ & $3500 \mathrm{~W}$ \\
Focal diameter $d_{f}(86 \%)$ & $36 \mu \mathrm{m}$ & $482 \mu \mathrm{m}$ \\
Rayleigh Length $z_{R}$ & $0.7 \mathrm{~mm}$ & $9.52 \mathrm{~mm}$ \\
Wavelength $\lambda$ & $1.07-1.08 \mu \mathrm{m}$ & $10.6 \mu \mathrm{m}$ \\
Diffraction coefficient $M^{2}$ & 1.38 & 1.81
\end{tabular}

Table 2. Parameter sets of the remote-laser processing of the open-hole specimens.

\begin{tabular}{ccccccc}
\hline Name & $\begin{array}{c}\text { Wavelength } \\
\text { in } \mu \mathrm{m}\end{array}$ & $\begin{array}{c}\text { Laser power } \\
\boldsymbol{P}_{\boldsymbol{L}} \text { in W }\end{array}$ & $\begin{array}{c}\text { Spotvelocity } \\
\boldsymbol{V}_{\boldsymbol{s}} \text { in } \mathrm{ms}^{-1}\end{array}$ & $\begin{array}{c}\text { Number of } \\
\text { cycles } \boldsymbol{n}\end{array}$ & $\begin{array}{c}\text { Focal position } \\
\Delta \boldsymbol{z} \text { in } \mathbf{m m}\end{array}$ & $\begin{array}{c}\text { Delay } \boldsymbol{t}_{\boldsymbol{p}} \\
\text { in } \boldsymbol{s}\end{array}$ \\
\hline $\mathrm{L} 12$ & $1.07-1.08$ & 1860 & 5 & 40 & -0.9 & 1 \\
$\mathrm{~L} 25$ & $1.07-1.08$ & 4650 & 1 & 4 & -0.9 & 1 \\
$\mathrm{CO}_{2}-14$ & 10.6 & 2870 & 0.5 & 7 & 0 & 2 \\
\hline
\end{tabular}


Table 3. Emission coefficient of the CFRP.

\begin{tabular}{cccc}
\hline Temperature & $\begin{array}{c}\text { Camera measuring } \\
\text { range }\end{array}$ & $\begin{array}{c}\text { Emission Coefficient } \\
\text { CFRP } \varepsilon\end{array}$ & $\begin{array}{c}\text { Transmission coefficient } \\
\text { Germanium window } \boldsymbol{\tau}\end{array}$ \\
\hline $22.7^{\circ} \mathrm{C}$ & $10^{\circ} \mathrm{C}-100^{\circ} \mathrm{C}$ & 1 & 1 \\
$100^{\circ} \mathrm{C}$ & $10^{\circ} \mathrm{C}-100^{\circ} \mathrm{C}$ & 0.64 & 0.39 \\
$150^{\circ} \mathrm{C}$ & $60^{\circ} \mathrm{C}-200^{\circ} \mathrm{C}$ & 0.64 & 0.39 \\
\hline
\end{tabular}

for the set temperature, the emission coefficient was adjusted for a thermographic image of a CFRP specimen. In this way the oven temperature and the displayed material temperature were matched. Since the radiation of the fiber laser is dangerous for the integrity of the sensor chip of the thermal camera, it must be shielded by a germanium window during the cutting trials. Its transmissivity was determined by comparing thermographic images taken with and without the window between the camera and the CFRP sample. Using the known transmittance of the germanium window, its influence on the temperature measurement can be compensated by the camera software.

The HAZ is defined as the area at a laser-cut edge that is influenced due to the thermal impact of the processing. A change in properties, even if reversible, for a fully cross-linked thermoset polymer first occurs when the glass transition temperature $T_{G}$ is reached. The resulting softening of the polymer can irreversibly release internal mechanical stresses resulting from the consolidation process of the fiber reinforced polymer. For this reason, the maximum distance of the isotherm of this temperature from the cut edge, which occurs during the cutting process, is considered as the expansion of the HAZ. However, it is not possible to register the expansion of the HAZ defined in this way by taking cross-sectional images. The material changes visible there are irreversible effects. On the one hand, this is a region within which the polymer matrix has evaporated, but the carbon fibers, which are significantly more temperature-stable, are still present. The MEZ is a part of the HAZ [10] [11]. On the other hand, changes in the carbon filaments are also visible. There is a swelling of the fiber ends that can be recognized [12] [13]. The extension of the MEZ at a single cut edge is not constant. One reason is the anisotropic thermal conductivity of a single laminate layer. Different fiber orientations within a composite thus lead to the deviations. It is therefore necessary to clearly define a procedure for obtaining the width of the MEZ. Since all tests are carried out on the same CFRP material, the method of determining the mean expansion of the MEZ is chosen [14]. Thus the measured values are comparable to each other. In this method, the width of the MEZ is determined by dividing its total area visible in the cross section by the material thickness.

\section{Results and Discussion}

\subsection{Determination of the MEZ in Dependency of the Remote Fiber-Laser Cutting Parameters}

For the laser power $\mathrm{P}_{\mathrm{L}}$ of the fiber laser, three levels were used in the variation to 
develop cutting parameter sets with the fiber laser system. These correspond to $40 \%, 70 \%$ and $100 \%$ of the maximum effective available laser power of $P_{L, \max }=$ $4650 \mathrm{~W}$. All stated laser powers are those measured in the working field. The optical beam transport losses are thus taken into account. Preliminary tests have shown that the number of exposure cycles required for the formation of a complete cut is not changed by varying the focus position between the upper and lower material surface. Since the Rayleigh length is smaller than the material thickness, the focus position was located in the materials center. As a result, the deviation of the laser intensities is the smallest both on the top and bottom of the material compared to the maximum intensity in focus. The values for the variation of the spot velocity $\mathrm{v}_{\mathrm{s}}$ were $1 \mathrm{~m} / \mathrm{s}, 3 \mathrm{~m} / \mathrm{s}$ and $5 \mathrm{~m} / \mathrm{s}$. For each combination of both parameters, the number of exposure cycles $n$ necessary to form a complete circular thru cut with a diameter of $4.8 \mathrm{~mm}$ was determined. For the measurement of the MEZ, straight cuts were made with the parameters determined for the circular holes. They were prepared into cross sections for a microscopic investigation. This avoids inaccuracies as the results do not depend on the exact position of the grinding plane as with a circular cut. Figure 2 shows exemplary microscopic images of the cross sections of the remote-laser cutting parameters L12 and L25. For the determination of the mean width of the MEZ, both of the cutting edges of one parameter set were considered.

Figure 3 and Figure 4 show the results of the parameter variation of the cutting tests. The diagrams illustrate the relationship between the number of exposure cycles required to form a complete thru cut and the cutting parameters.

The expansion of the MEZ resulting from the different cutting parameters is shown in the diagrams in Figure 5 and Figure 6.

It is noticeable that an increase of the spot velocity reduces the expansion of the MEZ significantly at constant laser powers. At the same time, the number of exposure cycles that is necessary for the formation of a complete thru cut increases. The rise of the laser power within the examined process window at
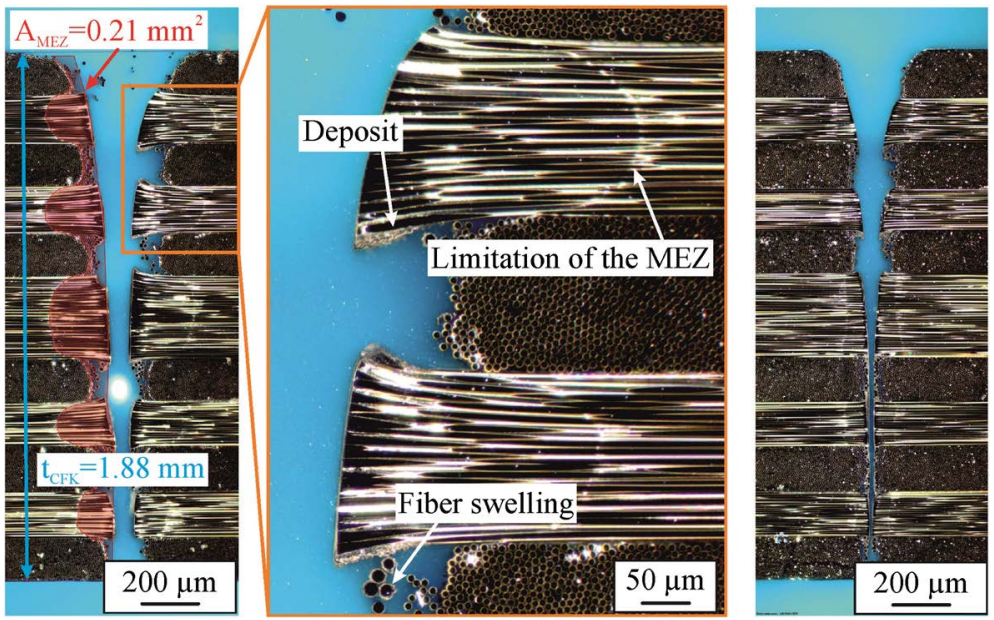

Figure 2. Microscoped cross sections of the remote-laser cutting parameters L25 with exemplary measurement of the MEZ (left) and in detail (mid) and L12 (right). 


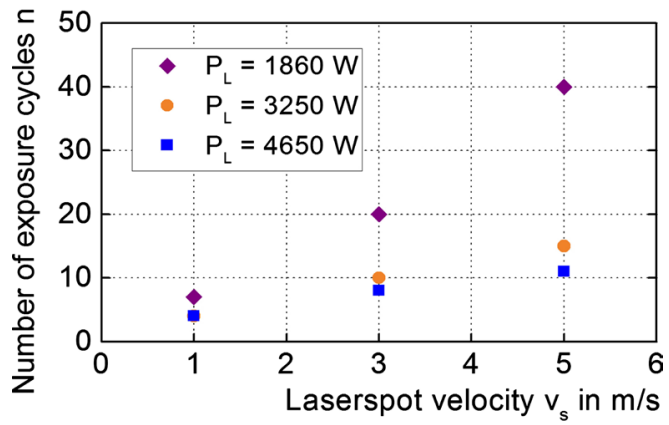

Figure 3. Number of exposure cycles in dependency of the spot velocity $V_{s}$.

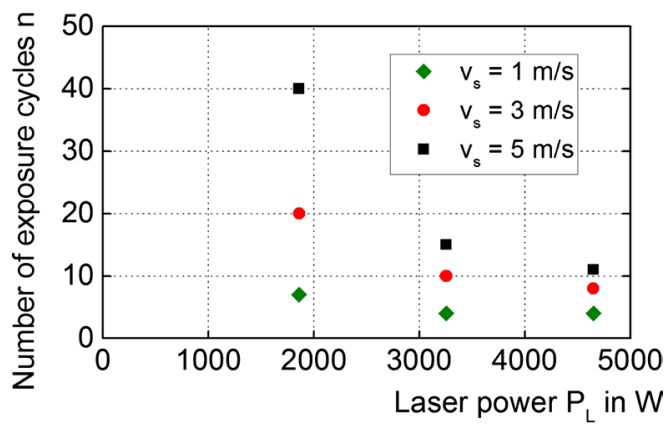

Figure 4. Number of exposure cycles in dependency of the laser power $P_{L}$.

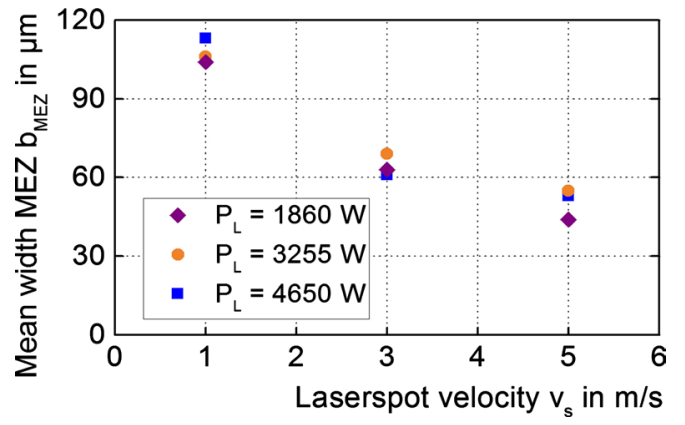

Figure 5. Mean width of the MEZ $b_{M E Z}$ in dependency of the spot velocity $v_{s}$.

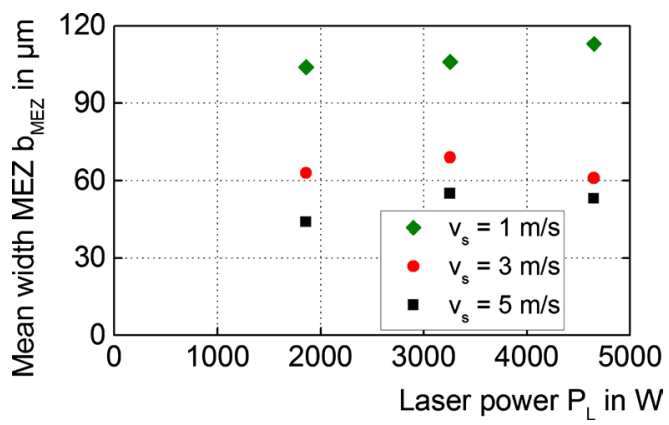

Figure 6. Mean width of the MEZ $b_{M E Z}$ in dependency of the laser power $P_{L}$.

constant spot velocities leads only to a small increase of the MEZ, but is able to reduce the number of exposure cycles considerably, especially at high spot velocities. This means that the productivity of the process is scalable with the applied laser power without excessively promoting thermal material damage. In contrast, 
increasing the spot velocity at constant laser powers does not lead to a higher productivity of the process. Within the observed parameter window there is an approximately linear relationship with the exposure cycle number. It also can be seen that there is an inversely proportional relationship between the laser power and the number of exposure cycles as well as between the spot velocity and the mean width of the MEZ.

In conclusion, the use of ever powerful and more expensive laser beam sources thus continuously brings smaller advantages with regard to the number of exposure cycles. In addition, the laser power that is transportable by the optical elements is limited. The situation is similar to the spot velocity. Their increase leads to smaller and smaller advantages with regard to a narrow expansion of the MEZ. Ultimately, it can therefore be assumed that an economical laser cutting process of CFRP is in principle associated with the formation of a MEZ and a HAZ. This requires a fundamental understanding of their effect on material properties, including mechanical behavior.

\subsection{Quasistatic and Cyclic Tensile Testing of Milled and Remote-Laser Cut Open-Hole Specimens}

The influence of the HAZ on the mechanical properties was investigated with quasi-static and cyclic experiments. In order to examine the influence of an excessively extended $\mathrm{HAZ}$, a $\mathrm{CO}_{2}$ laser system was used for the quasi-static investigations in addition to the fiber laser. Although the CFRP has a high absorption for the wavelength of $10.6 \mu \mathrm{m}$, the use of this system leads to the formation of a wide HAZ [15]. The longer wavelength of the radiation and the large focal length of the scanner result in a significantly larger spot diameter compared to the fiber laser (Table 1). For this reason the intensity is lower. Therefore smaller spot velocities are necessary to form a cut. The resulting increase in the interaction time between the laser and the CFRP results in the wider HAZ.

The highest tensile stress that occurs in the quasi-static test is defined as $\sigma_{\max }$. The definition of the maximum stress is given in Figure 7 exemplary. As a result of the quasi-static tension tests, the maximum stresses $\sigma_{\max }$ are shown in the diagram in Figure 8. They are given in dependency of the mean extension of the MEZ. On the one hand side, the results of initial quasistatic tensile tests are reported in the diagram. They have been performed to find the stress horizons for the cyclic fatigue tests. On the other hand side, the maximum stresses of cyclic tests are shown, since single specimens failed at the first load cycle.

A trend of increasing maximum stresses become obvious up to certain extend. The reason is the redistribution of mechanical shear stresses because of the thermal degradation of the CFRP at the cutting edge. These lead to a reduction of the notch stress concentrations [6] [7]. However, this trend is reversed with a further growth of the MEZ, as the present results indicate. The reason for this can be increasing damage to the load-bearing fibers. These oxidize at elevated temperatures [16] [17]. Due to this, they lose their strength. This is primarily not due to a decrease in the cross-sectional area of the fibers, but the formation of surface defects 
on the single filaments [17]. Because of the increasing expansion of the MEZ, the number of filaments damaged in this way increases. This effect more and more superimposes the advantageous reduction of the notch stress concentration.

The standard deviation of the maximum mechanical stresses of each of the four test series is not more than $4 \%$. The reasons for the scatter are material defects resulting from the production of the CFRP sheet material. These can be local deviations in the alignment of individual filaments or fiber bundles, for example. Another influence of the manufacturing process can be single voids in the polymer matrix, which are a starting point for inter fiber breaks.

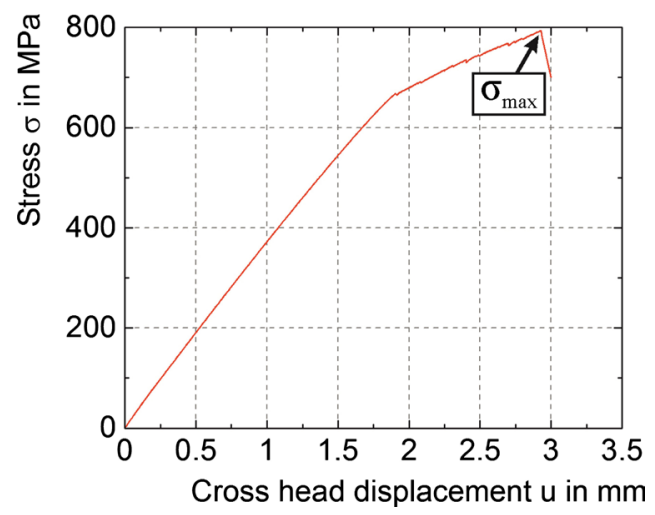

Figure 7. Definition of the maximum stress $\sigma_{\max }$ at an exemplary stress-displacement plot of the specimen ES-L25-5.

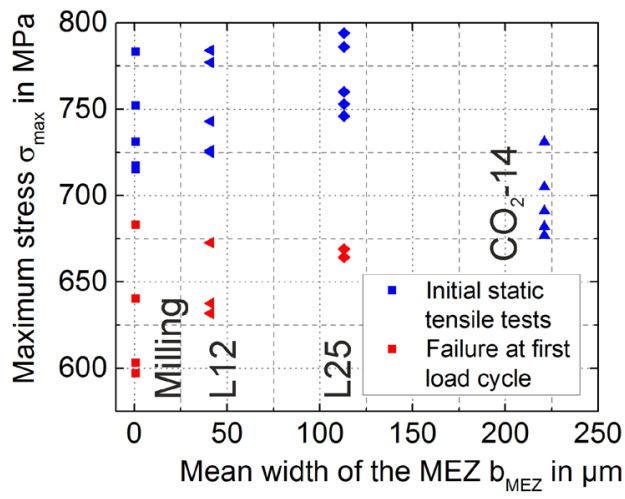

Figure 8. The quasi-static maximum stresses of the open-hole specimens in dependency of the width of the MEZ.

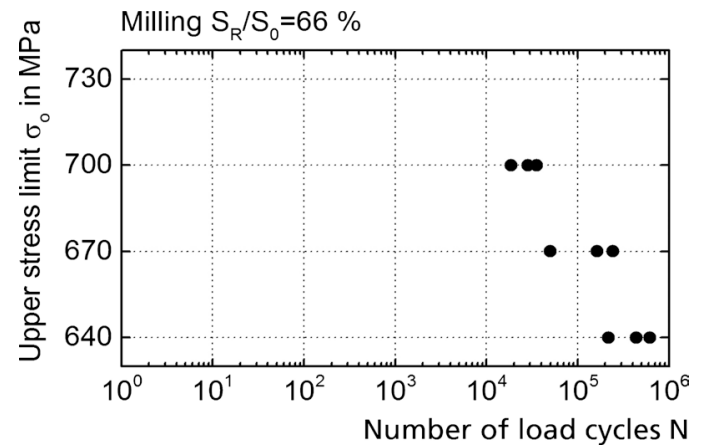

Figure 9. S-N Plot for a relative residual stiffness $S_{R} / S_{0}$ of $66 \%$ of milled specimens. 


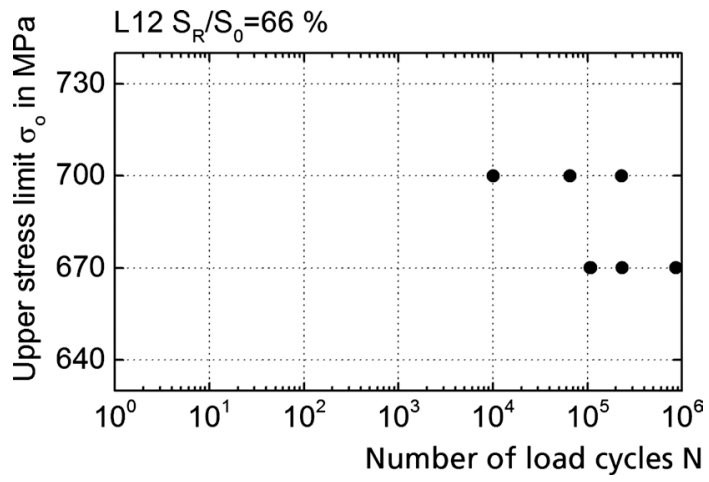

Figure 10. S-N Plot for a relative residual stiffness $S_{R} / S_{0}$ of $66 \%$ of remote-laser parameter L12 cut specimens.

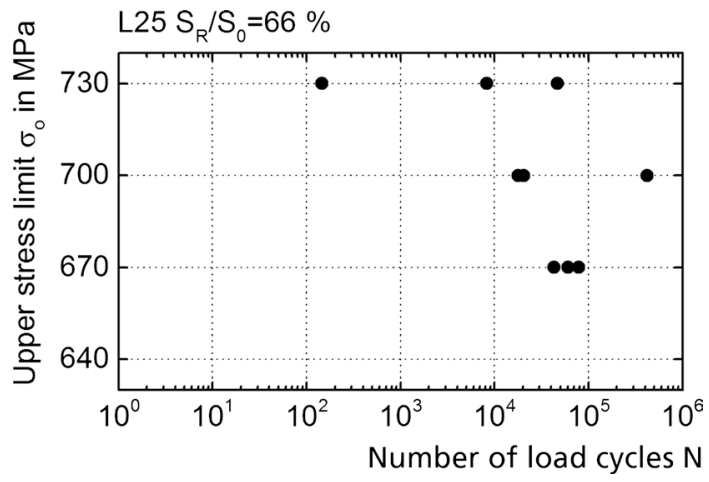

Figure 11. S-N Plot for a relative residual stiffness $S_{R} / S_{0}$ of $66 \%$ of remote-laser parameter L25 cut specimens.

The fatigue tests reveal that the open-hole specimens of the bidirectional CFRP which survive the first load cycle, are predominantly failsafe up to $10^{6}$ load cycles. This is even for the highest load horizons investigated $\left(\sigma_{o} / \sigma_{B} \approx 0.95\right)$. Figure 9, Figure 10 and Figure 11 show S-N plots from the tests on the open-hole specimens. In the diagrams, the upper stress limits $\sigma_{o}$ are assigned to those load cycle numbers $\mathrm{N}$ at which the test specimens achieve a residual stiffness $S_{R} / S_{0}=$ $66 \%$. For milling and remote-laser beam cutting with the parameter L12, similar degradation behaviors are shown. The reaching of the defined relative residual stiffness limit $S_{R} / S_{0}=66 \%$ for the laser cutting process is shifted to some higher load cycle numbers on average. However, the scattering of the individual measurement results clearly increased. For an upper stress limit of $\sigma_{o}=700 \mathrm{MPa}$, the average number of load cycles of the remote-laser cutting parameter L25 is also comparable with these results. Here, for the lower upper stress limit $\sigma_{o}=670$ $\mathrm{MPa}$, however, no increase in the number of load cycles $\mathrm{N}$ can be determined. The results of damage evolution, which are phenomenologically measured by means of residual stiffness, are influenced by the materials initial state. In addition to the potential pre-damages introduced by the remote-laser cutting process, this also includes the statistically distributed material imperfections from the production of the CFRP material described above. These effects superimpose each other. Therefore, the remarkably low number of load cycles of the 
parameter set L25 at the stress horizon of $\sigma_{o}=670 \mathrm{MPa}$ cannot be used to conclude a general influence of remote-laser beam cutting. This would require a statistical validation through more experiments, which go beyond the three available tests of each stress horizon. Nevertheless, the results of the parameter set L12 in particular show that the remote-laser cutting process does not produce such pre-damage, which causes an increased stiffness degradation compared to milling. A precondition for this is an appropriate design of the remote-laser process.

The diagram in Figure 12 also indicates this. It shows the relative residual stiffness $S_{R} / S_{0}$ for all stress horizons after $N=10^{6}$ load cycles. There are no noticeable differences between the residual stiffnesses of the milled and the remote-laser cut samples of both of the parameter sets. At the horizon of an upper stress limit of $\sigma_{o}=730 \mathrm{MPa}$ two of the three tested specimens failed before they reached $10^{6}$ load cycles.

\subsection{Thermography of the Laser Cutting Process as an Approach to Process Control and Monitoring}

The mechanical tests have shown that remote-laser cutting can be advantageous for the load bearing capacity of notched CFRP components in particular under quasi-static load conditions. However, an excessive expansion of the MEZ leads to a loss of this benefit. In order to guarantee the required mechanical properties even under cyclic loading conditions, it is necessary to ensure that the permissible degree of thermal damage is maintained. The expansion of the HAZ can be influenced by the process parameters. Due to the sensitivity of the mechanical properties of a notched component to this factor, process monitoring is useful in the manufacturing of components. It allows quality assurance and can serve as one aspect of proof of faultless production. In the following the suitability of process thermography for this purpose is examined.

The determination of isotherms was tested as an approach to evaluate the thermography data. It should be noted that the temperatures that can be measured on the surface are not equal to those inside the material [18]. First of all, the characteristic temperatures of the CFRP were determined. A thermogravimetric analysis has shown that the thermal matrix decomposition starts at about $T_{Z}=$

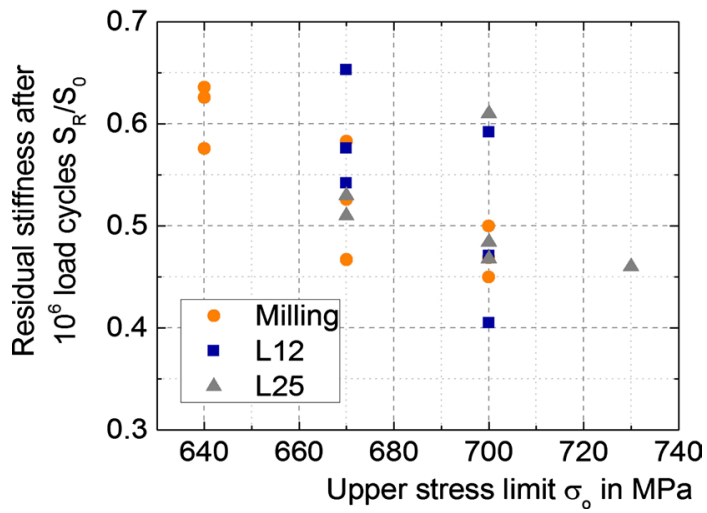

Figure 12. Relative residual stiffness $S_{R} / S_{0}$ after $10^{6}$ load cycles. 
$350^{\circ} \mathrm{C}$. The glass transition temperature of the epoxy according to the manufacturer's data sheet is $T_{G}=140^{\circ} \mathrm{C}$. During an exposure cycle of the laser beam, the vaporized material emerges from the kerf, as shown in Figure 13. This hot gas covers the process zone, which is why the material temperatures cannot be measured at this point in time. After the extraction of the waste gas it becomes obvious that the cooling rates are very high. They do not allow the determination of the isotherms of the initial matrix decomposition temperature (Figure 14). A reliable and precise process monitoring with the aim to control the width of the MEZ directly by measuring its isotherm $\mathrm{T}_{\mathrm{Z}}$ is not possible under the given restraints.

In contrast to the isotherms of the initial decomposition temperature, those of the glass transition can be determined reliably. Due to the heat conduction, they have the maximum distance to the cutting kerf after the hot waste gas has been extracted (Figure 15). The remote-laser cutting parameters L25 and L12 show the largest extension of the temperature range within which the glass transition temperature is exceeded, as it can be seen in the diagram in Figure 16. In contrast, parameter L12 causes the MEZ with the smallest expansion. It is not possible to predict the extent of the HAZ using this method within the examined field of parameters reliably. The reason for the deviations can be the heat accumulation that occurs at the edge of the cut due to the high number of exposure cycles

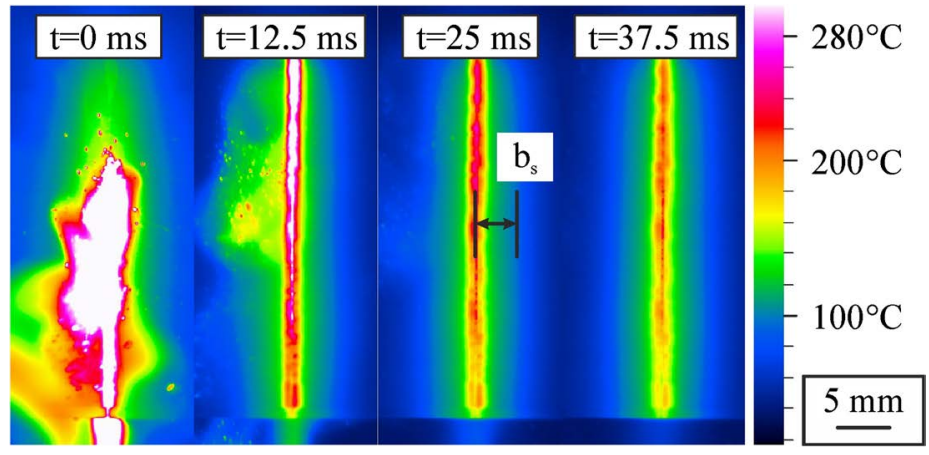

Figure 13. Single frame sequence of the last exposure cycle of the remote-laser cutting parameter L17.

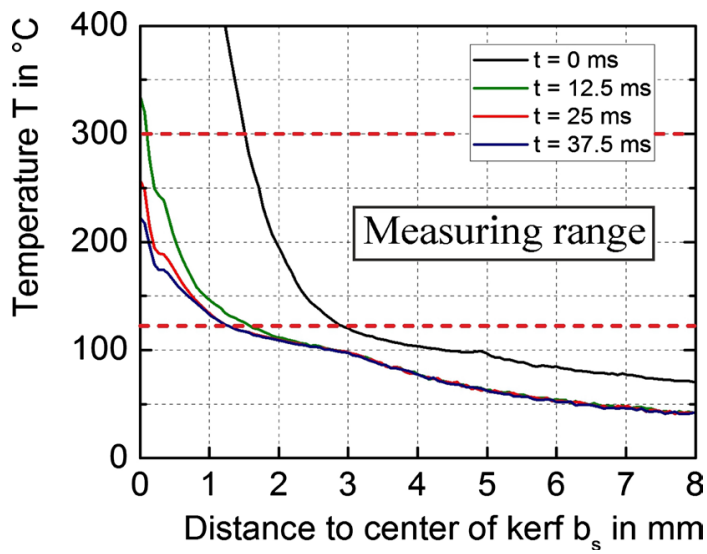

Figure 14. Time sequence of the temperature curves of the last exposure cycle of parameter L17. 
of $n=40$. The hot gas escaping from the kerf interacts with the materials surface. It heats the area near the cutting kerf in addition to the heat conduction [18]. This leads to a widening of the surface temperature field. Nevertheless, the method can be used for quality assurance. The maximum expansion of the isotherm of the glass transition can be recorded after each exposure cycle and compared with a known nominal value for the respective parameter set.

The diagram in Figure 17 shows another methodology to thermography-based process monitoring. There, the maximum temperature in the measuring field as a function of time is plotted. The diagram in Figure 18 is derived from these maximum temperature plots. The temperature value $\Delta T_{D, m}^{100}$ shown there is the average temperature difference between the turning points of consecutive exposure cycles

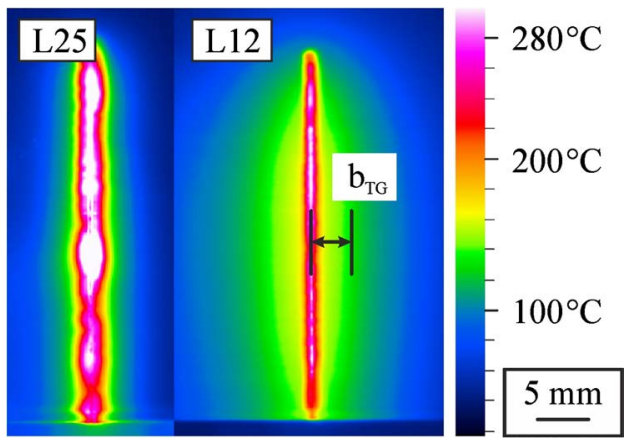

Figure 15. False color plot of temperature distribution immediately after the last exposure cycle.

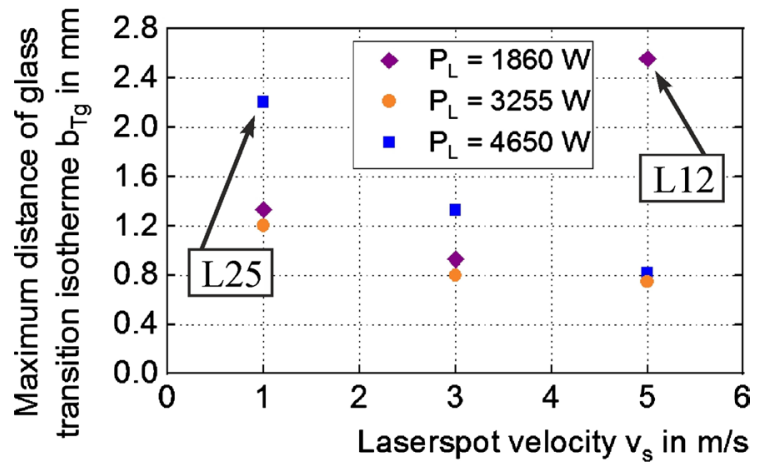

Figure 16. Maximum distance of the isotherm of the glass transition temperature $b_{\mathrm{Tg}}$ from the center of the kerf.

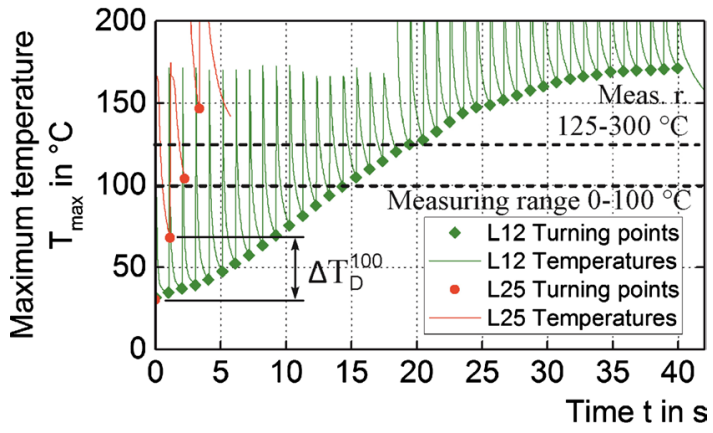

Figure 17. Maximum temperatures for remote-laser beam cutting with parameter L12 and L25. 


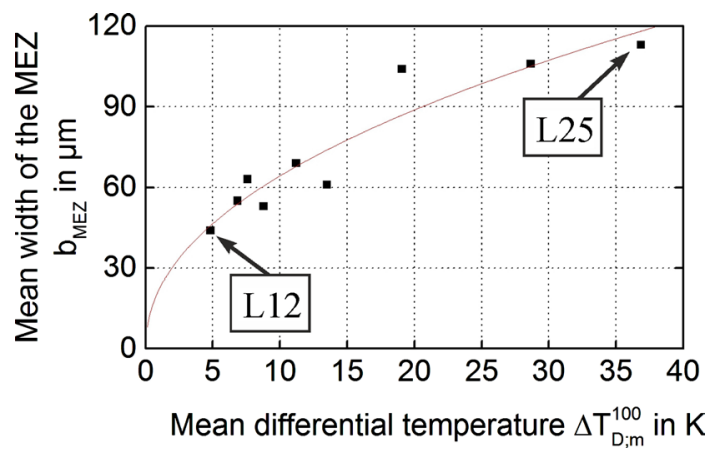

Figure 18. Expansion of the MEZ depending on the average temperature difference of the maximum temperatures before the exposure cycles.

$\Delta T_{D}^{100}$. The mean values of the temperature differences $\Delta T_{D}^{100}$ in the measuring range up to $100^{\circ} \mathrm{C}$ are plotted, since thermal equilibrium can occur at higher temperatures and a high number of exposure cycles. In dependency of $\Delta T_{D, m}^{100}$, the averaged widths of the MEZ of the different parameter sets are plotted. It can be seen that its widths increase with an increasing $\Delta T_{D, m}^{100}$.

Using this method it is therefore also possible to ensure quality control and process monitoring. Processing errors that affect the expansion of the MEZ can be registered by a deviation of the differential temperature from the setpoint for each laser exposure cycle. If temperature equilibrium is established at high numbers of exposure cycles, a setpoint value can also be used for control.

\section{Conclusions}

The remote-laser cutting tests with continuously emitting beam sources have shown that the formation of a MEZ at the cutting edge cannot be avoided. However, by adjusting the cutting parameters it is possible to influence their expansion. Quasi-static tensile tests showed that the failure stress of open-hole specimens is sensitive to the expansion of the HAZ, which was indirectly characterized by measuring the MEZ. This is indicated by an increase in the maximum stress with a growing MEZ, up to a certain extend. This means that in the case of remote-laser-cut mechanical notches, the presence of a HAZ can be advantageous. However, its excessive expansion reduces the load-bearing capacity of a component machined in this way. In addition, cyclic mechanical tests have shown that test samples processed by remote-laser beam cutting do not show greater degradation than those processed by milling. Every process for the production and consolidation of fiber reinforced composites leads to statistically distributed material defects. These material imperfections influence the mechanical properties of the material and are also subject to a statistical behavior which is characterized by a pronounced scattering, especially in the case of fatigue. However, an influence of the thermal damage of remote laser beam cutting on the cyclic degradation, which goes beyond the influence of material imperfections from the consolidation, could not be proven.

Basically, together with a suitable process control, laser beam cutting with a 
remote laser beam does not lead to disadvantages compared to milling. The remote-laser processing parameters have an influence on the expansion of the MEZ and thus also on the mechanical component properties. Process monitoring is therefore necessary to be able to prove the required component properties. The investigations show that process thermography is suitable for this application. Both the detection of the isotherms of the glass transition and the temperature difference between consecutive exposure cycles are suitable strategies.

The present results of the fatigue tests aim at evaluating the effect of the damage evolution as a consequence of remote laser cutting CFRP. However, for a better statistical validation the data base has to be extended by additional tests. Further investigations must surpass the present still rather phenomenological approach to what extent the cutting process has an influence on the proper mechanical damage initiation. It should be investigated in which way the presence of a HAZ promotes or delays the formation of cracks. This requires tests at smaller load horizons than the present ones, which do not overload the matrix-dominated $90^{\circ}$ individual layers. Moreover, the formation and propagation of inter-fiber breaks due to fatigue can be observed with appropriate in-situ methods, which should also be part of future research work.

\section{Acknowledgements}

The authors gratefully thank the German Research Foundation (DFG) for the financial support of the project 372786 314: "Analyse der Korrelation zwischen dem Ermüdungsverhalten Remote-Lasergeschnittener Faserkunststoffverbunde und der Prozessführung" (PI: Prof. Zimmermann/Prof. Kästner, TUD).

\section{Conflicts of Interest}

The authors declare no conflicts of interest regarding the publication of this paper.

\section{References}

[1] Klotzbach, A., Hauser, M. and Beyer, E. (2011) Laser Cutting of Carbon Fiber Reinforced Polymers using Highly Brilliant Laser Beam Sources. Physics Procedia, 12, 572-577. https://doi.org/10.1016/j.phpro.2011.03.072

[2] Harada, Y., Kawai, K., Suzuki, T. and Teramoto, T. (2012) Evaluation of Cutting Process on the Tensile and Fatigue Strength of CFRP Composites. Materials Science Forum, 706-709, 649-654. https://doi.org/10.4028/www.scientific.net/MSF.706-709.649

[3] Herzog, D., Jaeschke, P., Meier, O. and Haferkamp, H. (2008) Investigations on the Thermal Effect Caused by Laser Cutting with Respect to Static Strength of CFRP. International Journal of Machine Tools and Manufacture, 48, 1464-1473. https://doi.org/10.1016/j.ijmachtools.2008.04.007

[4] Harada, Y., Suzuki, T., Nishino, M. and Niino, H. (2012) Investigation on the Tensile Strength of CFRP/CFRTP Manufacturing Using High-Power Lasers. Proceeding of International Symposium on Laser Processing of CFRP and Composite Materials, Yokohama, 26-27 April 2012. 
[5] Stock, J.W., Zaeh, M.F. and Spaeth, J.P. (2014) Remote-Laser Cutting of CFRP: Influence of the Edge Quality on Fatigue Strength. SPIE 8963, High-Power Laser Materials Processing. Lasers, Beam Delivery, Diagnostics, and Applications III, 89630T. https://doi.org/10.1117/12.2037793

[6] Zaeh, M.F., Byrne, G. and Stock, J.W. (2017) Peak Stress Reduction in the Laser Contouring of CFRP. CIRP Annals-Manufacturing Technology, 66, 249-252.

https://doi.org/10.1016/j.cirp.2017.04.126

[7] Stock, J. and Kerschreiter, J. (2016) Modelling the Stress Concentration in CFRP at Notches with a Thermally Influenced Cut Edge. Advanced Materials Research, 1140, 288-295. https://doi.org/10.4028/www.scientific.net/AMR.1140.288

[8] Leone, C. and Genna, S. (2018) Heat Affected Zone Extension in Pulsed Nd:YAG Laser Cutting of CFRP. Composites Part B, 140, 174-182.

https://doi.org/10.1016/j.compositesb.2017.12.028

[9] Kalyanasundaram, D., Gururaja, S., Prabhune, P. and Singh, D. (2018) Open Hole Fatigue Testing of Laser Machined MD-CFRPs. Composites Part A, 111, 33-41. https://doi.org/10.1016/j.compositesa.2018.05.005

[10] Kononenko, T., Freitag, C., Komlenok, M., Weber, R., Graf, T. and Konov, V. (2018) Heat Accumulation between Scans during Multi-Pass Cutting of Carbon Fiber Reinforced Plastics. Applied Physics A, 124, 1-7. https://doi.org/10.1007/s00339-018-1647-9

[11] Freitag, C., Onuseit, V., Weber, R. and Graf, T. (2012) High-Speed Observation of the Heat Flow in CFRP during Laser Processing. Physics Procedia, 39, 171-178. https://doi.org/10.1016/j.phpro.2012.10.027

[12] Cheng, C., Tsui, Y. and Clyne, T. (1998) Application of a Three-Dimensional Heat Flow Model to Treat Laser Drilling of Carbon Fibre Composites. Acta Materialia, 46, 4273-4285. https://doi.org/10.1016/S1359-6454(98)00090-1

[13] Li, M., Li, S., Yang, X., Zhang, Y. and Liang, Z. (2019) Effect of Lay-Up Configuration and Processing Parameters on Surface Quality during Fiber Laser Cutting of CFRP Laminates. The International Journal of Advanced Manufacturing Technology, 100, 623-635. https://doi.org/10.1007/s00170-018-2728-9

[14] Jaeschke, P., Kern, M., Stute, U., Kracht, D. and Haferkamp, H. (2014) Laser Processing of Continuous Carbon Fibre Reinforced Polyphenylene Sulphide Organic Sheets-Correlation of Process Parameters and Reduction in Static Tensile Strength Properties. Journal of Thermoplastic Composite Materials, 27, 324-337. https://doi.org/10.1177/0892705712446016

[15] Fürst, A., Klotzbach, A., Hühne, S., Hauptmann, J. and Beyer, E. (2013) Remote Laser Processing of Composite Materials with Different Opto-Thermic Properties. Physics Procedia, 41, 389-398. https://doi.org/10.1016/j.phpro.2013.03.092

[16] Shayed, M.A., Cherif, C., Hund, R., Cheng, T. and Osterod, F. (2010) Carbon and Glass Fibers Modified by Polysilazane Based Thermal Resistant Coating. Textile Research Journal, 80, 1118-1128. https://doi.org/10.1177/0040517509357648

[17] Bertran, X., Labrugère, C., Dourges, M. and Rebillat, A. (2013) Oxidation Behavior of PAN-Based Carbon Fibers and the Effect on Mechanical Properties. Oxidation of Metals, 80, 299-309. https://doi.org/10.1007/s11085-013-9388-9

[18] Bluemel, S., Staehr, R., Jaeschke, P., Suttmann, O. and Overmeyer, L. (2015) Correlation of Internal and Surface Temperatures during Laser Cutting of Epoxy-Based Carbon Fibre Reinforced Plastics. Journal of Reinforced Plastics and Composites, 34, 662-671. https://doi.org/10.1177/0731684415576981 